\title{
Relationships between sleep paralysis and sleep quality: current insights
}

This article was published in the following Dove Press journal:

Nature and Science of Sleep

\author{
Dan Denis ${ }^{1,2}$ \\ 'Department of Psychiatry, Beth Israel \\ Deaconess Medical Center, Boston, \\ MA, USA; ${ }^{2}$ Department of Psychiatry, \\ Harvard Medical School, Boston, MA, \\ USA
}

\begin{abstract}
Sleep paralysis is the unusual experience of waking up in the night without the ability to move. Currently little is known about the experience, despite the fact that the vast majority of episodes are associated with extreme fear and in a minority of cases can lead to clinically significant levels of distress. The aim of this work was to review the existing literature pertaining to the relationship sleep paralysis has to sleep more generally, measured both with subjective questionnaires and objective laboratory recordings. In terms of subjective sleep variables, worse sleep quality has been found in multiple studies to be associated with increased odds of sleep paralysis occurrence. In addition, insomnia symptoms (but not a diagnosed insomnia disorder) have also been found to predict sleep paralysis. Associations between sleep paralysis and other unusual and/or threatening sleep experiences such as nightmares, exploding head syndrome, and lucid dreaming have been reported. In terms of objective measurements, the limited literature to date shows sleep paralysis to be a "mixed" state of consciousness, combining elements of rapid eye movement sleep with elements of wakefulness. Future research needs to focus on longitudinal designs to disentangle the direction of effects and more typically employ a broader assessment of sleep paralysis that better captures associated features such as hallucinations, fear, and distress.
\end{abstract}

Keywords: parasomnia, incubus, hypnagogic, hypnopompic

\section{Introduction}

Sleep paralysis involves a period of time at either sleep onset or upon awakening from sleep during which voluntary muscle movements are inhibited. Ocular and respiratory movements remain unaltered, and perception of the immediate environment is clear. ${ }^{1}$ Sleep paralysis is most closely linked to rapid eye movement (REM) sleep, as opposed to non-REM (NREM) sleep. REM sleep is associated with vivid dreaming. Throughout periods of REM sleep, there is total muscle atonia (save the eyes and respiratory system). ${ }^{2}$ This paralysis is a natural feature of normal REM sleep, possibly driven by GABA and glycine inhibition of motor neurons. ${ }^{3}$ One likely reason for this muscle paralysis is to prevent unwanted and dangerous movements from occurring during dreaming, when the cerebral cortex including motor cortex is active. ${ }^{4,5}$ The state of sleep paralysis is thus believed to arise from an ongoing continuation of the REM-induced muscle paralysis into a waking state. ${ }^{6}$

In addition to the ongoing muscle atonia, around $75 \%$ of sleep paralysis episodes are typically accompanied by a wide range of bizarre and often terrifying hallucinations. $^{7}$ These hallucinations typically fall into three categories. ${ }^{8,9}$ Intruder hallucinations consist of a sense of evil presence in the room, along with vivid multisensory 
hallucinations of a bedroom intruder. Incubus hallucinations describe a sense of pressure on the chest, often accompanied by sensations of being choked or suffocated. These two categories of hallucinations typically co-occur. ${ }^{8}$ The third category, vestibular-motor (V-M) hallucinations involve illusory feelings of movement, out-of-body feelings, and out-of-body autoscopy. ${ }^{9}$

It is possible that the hallucinatory content of sleep paralysis may result from the intrusion of REM-generated dream mentation into wakefulness. However, dream and sleep paralysis imagery differ greatly from one another. The vast majority (up to $90 \%$ ) of sleep paralysis episodes are associated with fear. ${ }^{8}$ This contrasts with $\sim 30 \%$ of dreams being rated as frightening. ${ }^{10}$ In a study comparing the contents of sleep paralysis and dream reports, sleep paralysis episodes were found to be emotionally uniform, feature more aggressive "characters," and the "dreamer" was more frequently the victim of attacks compared with typical dreams. ${ }^{11}$ Around $10 \%$ of those who experience sleep paralysis report that episodes lead to clinically significant levels of distress, and $7 \%$ report that sleep paralysis interferes with other aspects of their lives. ${ }^{12}$ Despite this not all episodes are fearful, with one survey finding that as many as $20 \%$ of those who experience sleep paralysis have had pleasant episodes. ${ }^{13}$

One of the primary differences between sleep paralysis and other states such as dreaming and nightmares is the fact that the individual is awake during the experience. Determining wakefulness is challenging however, especially when using subjective reports. Objective assessments of sleep paralysis have been able to confirm that the eyes do open during episodes and that brain activity shifts from REM sleep activity to a combined state containing both REM sleep and wake-like features (see the section: Objective sleep measurements for full discussion of this work). ${ }^{14,15}$ For subjective assessments it is more difficult, as episodes of sleep paralysis could be explained as a dream in which they believe they are awake, as opposed to actually being awake. Indeed, the phenomenon of false awakenings describes a very similar state. A false awakening is an experience in which individuals falsely believe that they have woken up, only to discover subsequently that the perceived awakening was part of a dream. Like sleep paralysis, false awakenings are reported to be highly realistic ${ }^{16}$ and can lead to anxiety in some cases. ${ }^{17}$

Prevalence estimates of lifetime episodes of sleep paralysis vary widely, with one systematic review suggesting a lifetime prevalence estimate of $7.6 \%$ (with individual study estimates ranging from $2 \%$ to $60 \%) .{ }^{18}$ In a meta-analysis focusing specifically on incubus experiences, a lifetime prevalence of $11 \%$ was found. ${ }^{19} \mathrm{~A}$ large number of variables have been associated with sleep paralysis..$^{20}$ In particular, sleep paralysis is more common in student and psychiatric samples. ${ }^{18}$ Specifically, patients with post-traumatic stress disorder and panic disorder have consistently been found to have elevated rates of sleep paralysis. ${ }^{21-26}$ In nonclinical samples, heightened levels of stress, exposure to potentially threatening events such as the death of a family member, anxiety, and general poor mental health all appear to be associated with the presence of episodes. ${ }^{20,21,27-29}$

Perhaps unsurprisingly, the presence and frequency of sleep paralysis share a close relationship with poor sleep quality more generally. Understanding the nature of this association is important both for furthering our basic understanding of sleep paralysis and for the purposes of developing treatments to reduce or even eliminate sleep paralysis in those who suffer worst from it. The aim of this article is to review the current literature on sleep paralysis and sleep quality. Studies looking at both subjective and objective aspects of sleep and its relationship to sleep paralysis will be reviewed. The presence of sleep paralysis in other sleep disorders will also be discussed.

\section{Linking components of subjective sleep quality to sleep paralysis}

Using otherwise healthy participants, a number of studies have found a link between subjectively rated sleep quality and occurrence of sleep paralysis. Using just a single item to assess sleep quality (How would you assess the quality of your sleep during the previous month?), one study in a large Chinese sample found that those who rated their sleep as either very good or good had significantly smaller odds of experiencing sleep paralysis than those who said their sleep was either very bad or bad..$^{30} \mathrm{~A}$ similar result was found in a Japanese sample that assessed sleep paralysis in over 90,000 participants. ${ }^{31}$

Sleep quality, however, is a multifaceted construct and can encompass many different factors such as sleep duration, sleep latency, as well as more subjective aspects such as the "restfulness" of sleep. As such, using a questionnaire that taps into these different factors is more insightful than asking a single question. A few studies have examined the relationship between occurrence of sleep paralysis and scores on questionnaire measures of sleep quality, such as the Pittsburgh Sleep Quality Index (PSQI). ${ }^{32}$ The PSQI is an 18-item questionnaire assessing seven components of sleep quality and disturbance over a 1-month period. The measure yields seven component 
scores (subjective sleep quality, sleep latency, sleep duration, habitual sleep efficiency, sleep disturbances, use of sleeping medication, and daytime dysfunction) as well as a global score, which is the sum of the seven components. ${ }^{32}$

In Chinese-Taiwanese patients with obstructive sleep apnea (OSA), sleep paralysis was significantly positively associated with global PSQI score. ${ }^{33}$ Similar results were found in a sample of Japanese students. ${ }^{34}$ A limitation of these two studies, however, was that it was not shown if sleep quality, as measured by global PSQI score, significantly predicted sleep paralysis independent of other known predictors of sleep paralysis such as anxiety symptoms. ${ }^{20}$ One study of sleep paralysis in UK participants examined a range of potential predictors for sleep paralysis, including sleep quality (as measured by PSQI global score), age and sex, anxiety symptoms, depressed mood, lifetime exposure to threatening events, alcohol and caffeine intake, and smoking behavior. ${ }^{27}$ Along with anxiety symptoms and lifetime exposure to threatening events, this study showed that poor sleep quality was an independent predictor of sleep paralysis in a multiple predictor model.

Only one study has examined which of the seven components of the PSQI best predicts sleep paralysis. ${ }^{35}$ Results of this work showed that longer sleep latency and increased daytime dysfunction were significantly associated with sleep paralysis. The sleep disruption component was also significant but did not survive multiple comparison correction. ${ }^{35}$ This suggests that sleep latency [how long it takes to fall asleep, assessed by the PSQI by the items "During the past month, how long (in minutes) does it usually take you to fall asleep at night" and "During the past month, how often have you had trouble sleeping because you cannot get to sleep within 30 minutes"] and daytime dysfunction (the impact bad sleep has on waking life, assessed by the items "During the past month, how often have you had trouble staying awake while driving, eating meals, or engaging in social activity" and "During the past month, how much of a problem has it been for you to keep up enough enthusiasm to get things done") are the most important aspects of sleep quality associated with sleep paralysis.

As well as general sleep quality, a few studies have also linked sleep paralysis to insomnia symptoms. Measuring symptoms of insomnia using Insomnia Symptoms Questionnaire, ${ }^{36}$ one study found a significant association between sleep paralysis and insomnia symptoms. ${ }^{35}$ Interestingly, this study found that when insomnia symptoms and global sleep quality were placed in the same regression model, only heightened insomnia symptoms independently predicted sleep paralysis.
This may suggest that symptoms of insomnia are a more important predictor of sleep paralysis than general sleep quality. In a different study using the Sleep Condition Indicator (SCI) to assess insomnia symptoms, ${ }^{37}$ total scores on the SCI significantly predicted the frequency of sleep paralysis independent of age, gender, daydreaming frequency and style, dissociative experiences, mindfulness, sensory imagery, symptoms of anxiety and depression, life stress, and paranormal and conspiracy beliefs. ${ }^{28}$ While increased insomnia symptoms also predicted increased frequency of intruder and incubus hallucinations, it was not associated with the intensity of hallucinations. ${ }^{28}$ Difficulty maintaining sleep was found to be a significant independent predictor of sleep paralysis occurrence in a sample of Chinese participants, whereas other insomnia symptoms such as difficulty initiating sleep and subjective insufficient sleep were not significant in a multivariate model (but were in univariate analyses).$^{38}$ One study found that self-reported insomnia (defined as waking up repeatedly during the night) occurring five or more times a month was associated with increased odds of experiencing sleep paralysis compared with experiencing insomnia less than five times a month. ${ }^{29}$ Variables associated with insomnia such as increased cognitive and somatic presleep arousal have been linked to sleep paralysis. ${ }^{35}$ Furthermore, faulty cognitions about sleep, such as being worried about losing control over one's own ability to sleep and being overwhelmed by thoughts at night and feeling that one has no control over these thoughts, has been associated with increased odds of sleep paralysis in one study. ${ }^{35}$

Given the close association between sleep paralysis and sleep quality, it is possible that they show significant genetic overlap. Recently, sleep and circadian rhythm genes have been implicated in sleep paralysis. Sleep paralysis has been shown to be heritable in twin studies, ${ }^{27}$ and variation in the PER2 gene (single nucleotide polymorphism rs2304672) has been shown to increase the odds of having experienced sleep paralysis. ${ }^{27}$ PER2 is a member of the Period (PER) family of genes and plays an important role in the regulation of circadian rhythms, such as the sleep-wake cycle. ${ }^{39,40} \mathrm{In}$ rodent studies, PER1/PER2 double knockout mice become totally arrhythmic in darkness, suggesting that these genes play an important role in keeping a consistent circadian cycle. ${ }^{39}$ It may be that dysregulation of the regular sleep-wake cycle increases the risk of experiencing sleep paralysis. ${ }^{41}$ An interesting future study would be to use multivariate genetic models to investigate whether similar genetic influences underlie both sleep paralysis and sleep quality.

As well as potential shared genetic influences, sleep quality may act as a mediator in the relationship between other 
stressors and sleep paralysis. Variables such as exposure to threatening events (eg, a death of a close friend or sibling, separation due to marital difficulties), life stress, and anxiety/ depression symptoms have been consistently linked to sleep paralysis ${ }^{20}$ and have also been associated with general sleep quality. ${ }^{42-44}$ An interesting hypothesis is that, for example, a period of heightened stress may cause a decline in general sleep quality, which in turn increases the risk of experiencing sleep paralysis. Unfortunately, direct testing of this claim has not been performed. This is due to the fact that no longitudinal studies of sleep paralysis have been conducted to date. One possibility would be to use existing large datasets and perform mediated regression analyses. ${ }^{45}$ This may help elucidate whether factors such as stress, depression, and anxiety are directly related to sleep paralysis or, as hypothesized here, they are related indirectly through first reducing sleep quality.

A reduction in sleep quality as a consequence of sleep paralysis should also be considered, especially in cases where sleep paralysis leads to significant levels of fear and postepisode distress. ${ }^{12,22}$ A number of factors have been shown to be linked to postepisode distress, including fear and threat/ assault experiences during sleep paralysis, general psychological stress, and supernatural beliefs about the nature of the episode. ${ }^{46}$ How postepisode distress relates to sleep quality has yet to be addressed. One possibility is that, following a fearful episode of sleep paralysis, sleep may be further disrupted by the individual trying to avoid sleep due to fear of sleep paralysis. Relatedly, the individual may lie awake at night before falling asleep worrying about having sleep paralysis again during the night. This idea is supported by one study, which found that dysfunctional beliefs about sleep and levels of presleep arousal were both predictive of sleep paralysis. ${ }^{35}$ Again, this was a cross-sectional study making it impossible to say whether these variables were a cause or consequence (or both) of sleep paralysis.

There is mixed evidence that factors such as bedtime and waking up time show any relationship with sleep paralysis. Two studies found that, with bedtime before 22:00 hours as a reference, going to bed between 22:00 and 00:00 hours was associated with reduced odds of sleep paralysis, while going to bed between 00:00 and 02:00 hours was associated with increased odds. ${ }^{30,31}$ Other research, however, has not found a link. ${ }^{34}$ With regard to waking up time, no relationship to sleep paralysis has been found. ${ }^{34}$ It is also unclear whether amount of sleep is associated with sleep paralysis, with research finding both longer and shorter durations predict sleep paralysis, as well as other studies finding no relationship. ${ }^{30,31,34,35,38}$ While overall sleep length may not be related to sleep paralysis, one study found that sleep paralysis episodes are most likely to occur in the beginning and middle of sleep compared with the end. ${ }^{47}$ Sleep paralysis most often occurs when lying in the supine position. However, sleeping position while falling asleep shows no relationship with sleep paralysis. ${ }^{47,48}$ Finally, sleeping in the same room/bed with a partner has been shown to not predict sleep paralysis. ${ }^{35}$

Other "anomalous" and/or potentially distressing sleep experiences appear to co-occur with sleep paralysis. For example, exploding head syndrome, the experience of hearing loud noises in one's head at either wake-sleep or sleep-wake transitions, ${ }^{1}$ has been associated with sleep paralysis in a couple of studies. ${ }^{49,50}$ Multiple studies have found a relationship between sleep paralysis and nightmare frequency. ${ }^{31,34,51}$ Disruptive nocturnal behaviors, which are sleep disturbances commonly associated with stress and trauma, ${ }^{52}$ also predicted the presence of sleep paralysis independent of general sleep quality in one study. ${ }^{35}$ In this context, disruptive nocturnal behaviors included phenomena such as nightmares of a traumatic experience, trouble sleeping due to general nervousness, and episodes of terror or screaming during sleep.

Anecdotal reports suggest that sleep paralysis and lucid dreaming are related. ${ }^{53} \mathrm{~A}$ lucid dream is a dream involving awareness of dreaming and is characterized by increased insight, control, access to waking memories, dissociation from one's own body, logical thought, and positive emotion, in contrast to nonlucid dreams. ${ }^{54,55}$ Intriguingly, some anecdotal accounts of sleep paralysis experiences suggest that it is possible to move from a sleep paralysis state into a lucid dream and vice versa. ${ }^{53}$ Systematic testing of this claim is still lacking; however, cross-sectional research has found a relationship. ${ }^{28,56}$ They showed that there was a significant correlation between frequency of sleep paralysis episodes and frequency of lucid dreams, based on self-report. Furthermore, one study found that lucid dreaming appeared to be specifically related only to those episodes of sleep paralysis that contained V-M hallucinations. Intruder/incubus type episodes were not significant predictors of lucid dreaming frequency. ${ }^{28}$ Other work using factor analysis methods on a wide range of unusual sleep experiences found experiences of intruder hallucinations fell on a different factor to lucid dreaming, also suggesting that lucid dreaming is not associated with intruder hallucinations. ${ }^{57}$ One possible explanation for this finding could be with control. Intruder experiences during sleep paralysis are consistently described as the individual having little to no control over the experience, such as the intruder hallucination is forcing the person to remain paralyzed or that the intruder is attacking the individual, and there is nothing 
they can do about it. Lucid dreaming is the clear opposite of this, as it is in part defined as being able to exert control over the dream. Anecdotal evidence suggests that V-M hallucinations can be controlled, are more positively valanced, and can be manipulated to induce out of body experiences, supposed "astral projection", and also as a way to enter lucid dreams. ${ }^{53}$ Systematic tests of these claims are a clear area for future research, as being able to gain control of sleep paralysis episodes to control the content may be a useful technique to disrupt episodes and turn them into positive experiences.

It has been proposed that the common co-occurrence of different anomalous sleep experiences may be due to dissociation. ${ }^{41,57}$ Dissociation is defined by the Diagnostic and Statistical Manual of Mental Disorder-V as "a disruption in the usually integrated function of consciousness, memory, identity, or perception of the environment." 58 Typically, the core features of dissociation are derealization (the feeling of disconnection from the world around you, eg, feeling like reality is a dream or movie) and depersonalization (the feeling of disconnection from oneself, eg, feeling as though you are separated from your body). Indeed, several studies have found sleep paralysis to be linked to levels of dissociative experiences, both in the context of severe trauma ${ }^{59,60}$ and in healthy samples. ${ }^{28,61}$ Furthermore, many studies have found levels of dissociation to be associated with a variety of sleep experiences such as bizarre dreams, hypnagogic hallucinations, and nightmares. ${ }^{41}$ Of note, the link between sleep and dissociation has not only been found in crosssectional questionnaire-based studies but a causal role of sleep disturbance in dissociative symptoms has been shown in studies of sleep deprivation, which found that the loss of sleep promoted increased dissociation. ${ }^{62}$ In a different longitudinal study, it was found that sleep normalization led to reduced symptoms of dissociation across a 6- to 8-week period. ${ }^{63}$ It should be noted that this study employed participants from an inpatient unit, who displayed a wide range of psychiatric and substance abuse disorders. The results of this work have led to the suggestion that individuals predisposed to a labile sleep-wake cycle (possibly due to genetic factors) promote the intrusions of sleep phenomena into waking consciousness, which may foster symptoms of dissociation. It is also possible that dissociative experiences lead to increased sleep disturbance, making a bidirectional relationship possible. ${ }^{41}$

\section{Objective sleep measurements}

Compared with the literature on subjective sleep variables, very little research on sleep paralysis using objective measures has been performed. This is at least partly due to the relative rarity of sleep paralysis, with it being particularly unusual to have individuals who experience sleep paralysis so frequently that they are likely to have an episode on any given night in a sleep laboratory. For example, in generally healthy samples, only $0.5 \%$ of participants report experiencing sleep paralysis multiple times a week. ${ }^{35}$

A couple of studies have used polysomnography (PSG), the gold standard for objective sleep recording, to identify potential differences in sleep architecture between those who report experiences of sleep paralysis compared with those who do not. In patients with OSA, no differences were found between patients also reporting sleep paralysis, compared with patients not reporting sleep paralysis, on any of the PSG measures reported. ${ }^{33}$ These measures were sleep efficiency (the percentage of time in bed that the individual was asleep), sleep latency (the number of minutes taken to fall asleep), REM sleep latency (the time taken to enter the first period of REM sleep), and percentage of the night spent in any particular stage (NREM stage 1, NREM stage 2, NREM stage 3, NREM stage 4, REM). ${ }^{33}$ This study was impressive in its sample size, with 41 participants with sleep paralysis and 66 without.

In a different study, potential objective sleep variables associated with sleep paralysis were investigated in 10 participants who reported between six episodes a year and one to three episodes a week and were otherwise healthy. They were compared with 10 narcolepsy patients and 10 healthy controls. ${ }^{64}$ This study found no differences between the three groups in sleep efficiency or percentage of times in all NREM sleep stages. Percentage of REM time was numerically lower in the sleep paralysis group compared with the narcolepsy and control groups (18.2\% vs $22.5 \%$ and $19.5 \%)$, but this difference was not significant. Sleep latency was found to be significantly longer in the sleep paralysis group (40.6 minutes) compared with the other two groups (narcolepsy: 19.5 minutes, controls: 19.2 minutes). In addition, REM latency was found to be longer in sleep paralysis participants (83.2 minutes) compared with the narcolepsy group (32.1 minutes) but not different from the healthy controls (98.7 minutes). ${ }^{64}$

From the very limited literature, little appears to be predictive of sleep paralysis in terms of objective sleep measurements, with the exception of long sleep latency found in one study. ${ }^{64}$ This finding has also been reported in studies investigating subjective sleep variables associated with sleep paralysis. ${ }^{35}$ The mechanism as to how longer sleep latency could be associated with sleep paralysis remains unclear, however, and warrants further research. One possibility is that the longer sleep latency is reflective of greater presleep 
arousal and heightened insomnia symptoms seen in those who have experienced sleep paralysis. ${ }^{35}$

Both of the above PSG studies did not attempt to record actual episodes of sleep paralysis in the laboratory environment. It is possible that in terms of general, trait-like sleep architecture, little distinguishes those who experience sleep paralysis from those who do not. It is likely, however, that on a night that sleep paralysis occurs, differences in PSG measures can be seen. A couple of studies have attempted to measure sleep paralysis episodes using PSG by eliciting sleep paralysis through interruption of the sleep cycle.

In the first study to attempt this, 16 participants who reported having at least two lifetime episodes of sleep paralysis spent seven consecutive nights in a sleep laboratory. ${ }^{15}$ Sleep was systematically disrupted in an attempt to induce an episode of sleep paralysis. Sleep was interrupted after 40 minutes of NREM sleep had elapsed from the termination of an REM period either in the first or third sleep cycle. After awakening due to the sleep interruption, participants performed an auditory vigilance task for 40 minutes. Following the task, participants went back to sleep and were awakened again after 5 minutes of REM sleep had elapsed. Following this awakening, participants were asked whether they had experienced sleep paralysis. From a total of 64 sleep interruptions, a total of six episodes of sleep paralysis were elicited (9.4\%). All six episodes occurred after participants went back to sleep following the vigilance task. All but one of the episodes occurred following a sleep-onset REM period (SOREMP), which is when a participant goes from being awake straight into REM sleep. The link between sleep paralysis and SOREMPs was supported in a subsequent study by the same authors. ${ }^{65}$ In this study, of 13 participants who self-reported at least two lifetime episodes of sleep paralysis, eight episodes of sleep paralysis were elicited from 184 sleep interruptions (4.3\%). As before, the majority of episodes occurred during/following a SOREMP (six/eight episodes). These two studies suggest a close relationship between sleep paralysis and REM sleep and particularly with unusual episodes of REM sleep that occur directly after wakefulness (Figure 1A).

PSG recordings of the episodes suggest that the state of sleep paralysis can be described as a "mixed" state of consciousness, blending elements of wakefulness, and REM sleep..$^{14,15}$ As shown in Figure 1B, EEG activity during the episodes showed abundant alpha activity being mixed with more typical REM activity. Alpha activity in the EEG is typically associated with quiet wakefulness and is normally absent during REM sleep. The mixed wake-REM sleep brain activity that was seen during sleep paralysis episodes occurred alongside continued muscle atonia, as shown by the flat-lined EMG signal throughout the episode. This indicates that the muscles are indeed paralyzed during episodes and likely represents a continuation of the normal atonia seen during REM sleep into a waking state of consciousness.

Additional evidence that sleep paralysis is a dissociated state, mixing wake and REM brain activity can be found from a case study of a single subject who's sleep paralysis episodes were recorded with PSG. ${ }^{66}$ They recorded EEG both from an eyes-closed waking state (when there is abundant alpha activity), a regular period of REM sleep, and a sleep paralysis episode. They then showed that statistically combining the waking and REM sleep periods together, the resulting activity was indistinguishable from the actual paralysis episode (Figure 1C). This finding, while only from a single participant, adds further evidence to the viewpoint that sleep paralysis is a mixture of waking and REM brain activity.

Theoretically, the conscious state of sleep paralysis can be modeled within the activation-input-modulation (AIM) framework, ${ }^{67,68}$ see also Figure 1D. The AIM model is a descriptive framework with three parameters within which different conscious states differ. ${ }^{69}$ Waking can be modeled as: 1 - a state of high neural activation; 2 - a dominance of external (environmental) over internal input; and 3 - high monoaminergic modulation. Normal REM sleep consists of: 1 - high activation, 2 - dominance of internal over external input, and 3 - cholinergic neuromodulation thought to account for the bizarre features of dream mentation. Sleep paralysis can be modeled as a mixture of these two states: 1 - high activation, 2 - mixed external and internal input, 3 - mixed neuromodulation. Direct evidence for both the activation and the input components has been shown. PSG evidence demonstrating sleep paralysis episodes containing REM and wake-like EEG activity confirms high neural activation. ${ }^{14,15,66}$ Mixed internal input is evidenced by the frequent reports of hallucinations that occur during sleep paralysis (internal input), while being awake and perceiving the immediate bedroom environment. It is on the input dimension that sleep paralysis differs from lucid dreaming. In former, there is a mixture of internal and external input, whereas the latter contains only internal input.

Brain regions/networks associated with sleep paralysis are currently unknown. During regular REM sleep, the stage of sleep most often associated with vivid dreaming, regions including medial prefrontal cortex, medial temporal lobe structures, motor cortex, and the anterior cingulate are activated, along with structures such as the pons/midbrain, caudate nucleus, and the amygdala. Conversely, dorso-lateral 


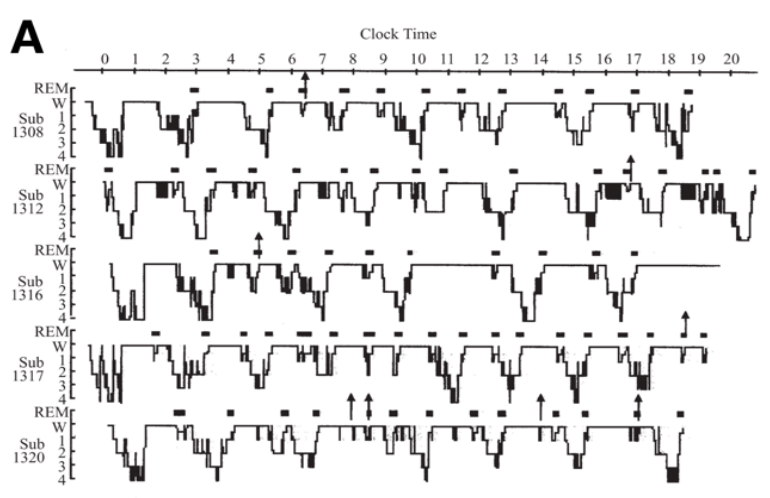

B
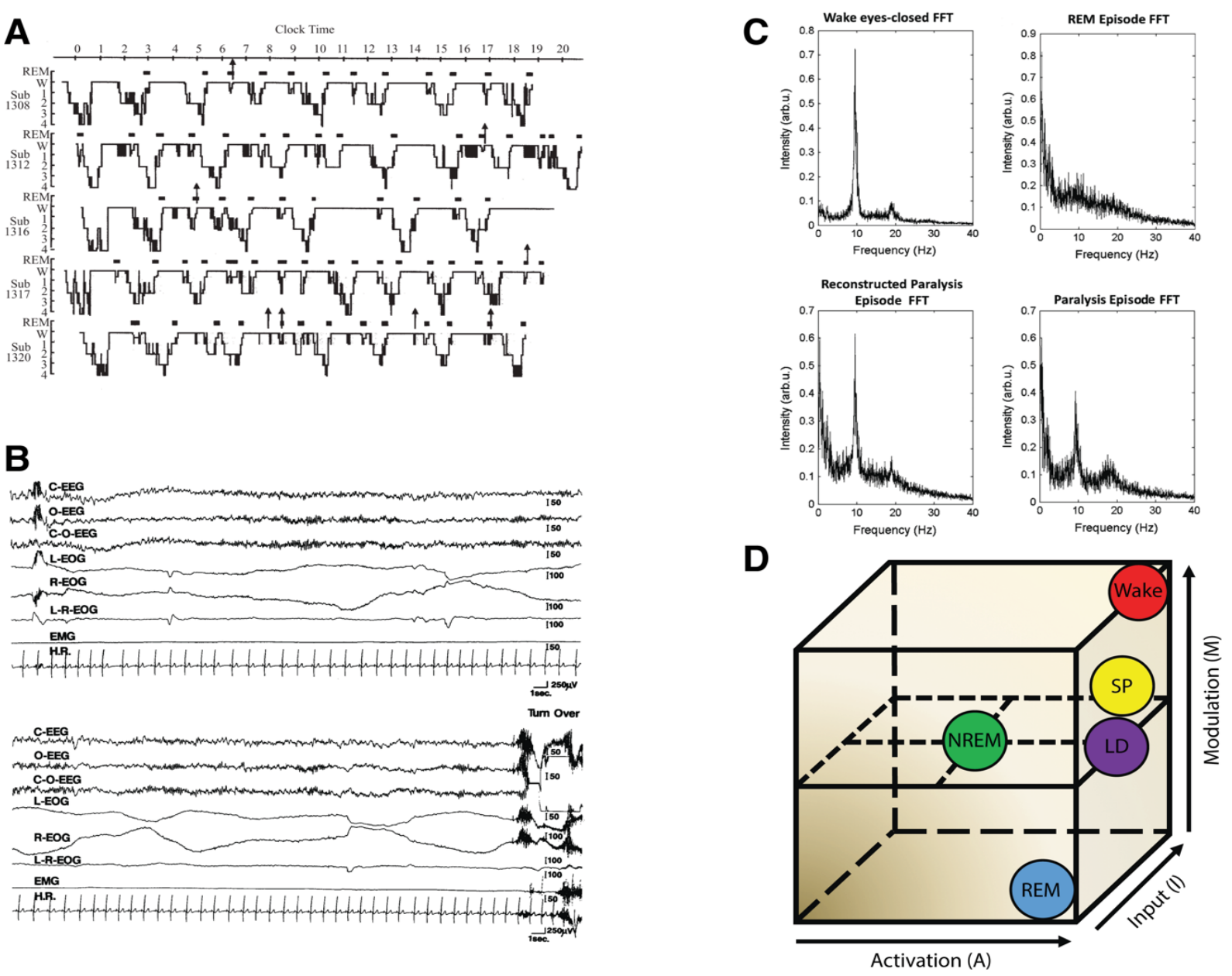

Figure I Physiology associated with SP.

Notes: (A) Hypnograms of five participants reporting SP. Each arrow indicates the awakening points when the participant reported SP. Note the close association between SP and REM sleep. Taken from the study by Takeuchi et al. ${ }^{65}$ (B) PSG recording during an SP episode. EEG shows REM activity mixed with abundant alpha. Both rapid and slow eye movements can be seen in the EOG channels. Muscle activity (measured by the EMG) remains flat throughout until the participant fully awakens at the end of the recording. Taken from the study by Takeuchi et al. ${ }^{15}$ (C) Spectral EEG data showing (clockwise from top-left) eyes closed waking, REM sleep, SP, and "reconstructed" SP by statistical combination of waking and REM sleep activity. Taken from the study by Terzaghi et al..$^{66}$ (D) The AIM state-space model. ${ }^{69} \mathrm{SP}$ is characterized by high activation, mixed internal/external input, and mixed neuromodulation.

Abbreviations: AIM, activation-input-modulation; FFT, fast Fourier transform; LD, lucid dreaming; NREM, nonrapid eye movement sleep; REM, rapid eye movement sleep; $\mathrm{SP}$, sleep paralysis.

prefrontal cortex, orbitofrontal cortex, and regions of the posterior cingulate cortex, inferior parietal cortex, and precuneus are deactivated relative wakefulness. ${ }^{5,70}$ Reactivation of some of these regions, particularly in frontal areas, has been shown to occur during lucid dreaming, explaining the increased metacognitive abilities found in this state. ${ }^{71-73}$

With regard to sleep paralysis, overactivation of the amygdala has been proposed to contribute to high levels of fear experienced during sensed presence/intruder hallucinations. ${ }^{8}$ The amygdala along with other limbic structures and the medial prefrontal cortex is involved in the recognition of processing of fear. ${ }^{74,75}$ Activation of these structures during REM sleep is believed to be related to the consolidation of emotional memories and fear extinction. Overactivation of the amygdala during REM sleep has been hypothesized to be involved in the generation of nightmares. ${ }^{76,77}$ In sleep paralysis, the individual becomes awake and conscious of their surroundings while still in a (partial) state of REM sleep. As such, fear-processing networks remain highly active, despite no immediate threat in the environment. This paradoxical state may then lead to the sense of an "evil" but unseen presence commonly reported during intruder-type sleep paralysis episode. Additionally, the fear associated with the sensation of paralysis, along with commonly reported breathing difficulties, is likely to increase fear-processing related activations even further. ${ }^{78}$

A different set of brain structures are likely to be implicated in V-M hallucinations. The posterior cingulate cortex 
has been shown to play an important role in the integration of self-location and perceived body ownership ${ }^{79}$ and is typically deactivated during REM sleep. Additionally, the temporo-parietal-junction (TPJ) has been hypothesized to play a role. ${ }^{80}$ In the context of out-of-body experiences more generally, disruption of TPJ processing has been argued to cause a failure to integrate vestibular, proprioceptive, tactile, and visual information relevant to the body. ${ }^{81,82}$ Given that pontine vestibular centers (which contribute to functions such as spatial orientation, movement, and balance) are closely associated with the pons, which is activate during REM sleep, and inferior parietal areas are deactivated, a multisensory integration failure seems a plausible explanation of V-M type hallucinations.

In summary, work on objective predictors and measurements of sleep paralysis are limited. From the few studies conducted to date, sleep latency and sudden-onset REM periods may be predictors of sleep paralysis occurrence. Of the studies that have recorded brain activity during sleep paralysis, results suggest sleep paralysis to be a dissociated state of consciousness, combining a mixture of waking and REM sleep brain states. Knowledge of brain regions and networks that activate during sleep paralysis are currently unknown; however, a number of potentially key areas have been proposed.

\section{Sleep paralysis in other sleep disorders \\ Narcolepsy}

Sleep paralysis is a common feature of narcolepsy, a neurological disorder afflicting $0.02 \%$ of the population. It is characterized by disturbed nocturnal sleep patterns, excessive daytime sleepiness, cataplexy, hypnagogic hallucinations, and sleep paralysis. Despite being removed as a diagnostic criteria in the most recent edition of the International Classification of Sleep Disorders, ${ }^{1}$ sleep paralysis is common in the disorder - being present in anywhere between $25 \%$ and $70 \%$ of cases. ${ }^{83}$ It is possible that sleep paralysis may share some physiological basis with cataplexy. Cataplexy is a sudden and uncontrollable muscle paralysis that occurs during wakefulness. It has been theorized that cataplexy represents the tendency for the occurrence of REM-related phenomena (namely muscle atonia) in wakefulness. ${ }^{84}$ Interestingly, hallucinations are more common in narcolepsy patients with cataplexy compared with narcolepsy without cataplexy. ${ }^{85}$ Furthermore, sleep paralysis is more common in narcolepsy with cataplexy patients (53\%) compared with narcolepsy without cataplexy $(32 \%) .{ }^{86}$
It is widely believed that the cause of narcolepsy is a deficiency in the hypothalamic neuropeptide hypocretin. ${ }^{87}$ In patients with low levels of cerebral spinal fluid (CSF) hypocretin-1, there is a higher rate of sleep paralysis $(52 \%)$ compared with patients with normal CSF hypocretin-1 $(33 \%){ }^{88}$ Despite differences in frequency of episodes, the severity of sleep paralysis did not differ between the two groups ${ }^{88}$ Hypocretin deficiency is believed to be an autoimmune response, ${ }^{87}$ with almost all narcolepsy with cataplexy patients testing positive for the human leukocyte antigen subtypes DR2/DRB1*1501 and DBQ1*0692. However, DR2/DBQ1 positivity has not been found to be linked to sleep paralysis. ${ }^{89,90}$ Overall, it appears that low CSF hypocretin-1 is linked with increased frequency of sleep paralysis. Although DR2/DBQ1 positivity has not been linked to the occurrence or severity of sleep paralysis, one of these studies used a very small sample size $(n=22) .{ }^{89}$ Future research is therefore warranted on this topic due to the small number of studies that have been performed to date.

\section{Idiopathic hypersomnia}

Like narcolepsy, idiopathic hypersomnia is a condition also featuring excessive daytime sleepiness, though cataplexy is not present. The diagnosis is tricky, as it requires the exclusion of other causes of excessive daytime sleepiness, which can be difficult to ascertain. ${ }^{91}$ One study found a high prevalence of sleep paralysis in hypersomnia patients (40\%), which was not statistically different from narcolepsy patients with (53\%) and without (32\%) cataplexy. ${ }^{86}$ Another study found sleep paralysis to be present in $\sim 27 \%$ of hypersomnia patients. ${ }^{92}$ When comparing patients with and without a long-sleep time, no difference in sleep paralysis prevalence was found..$^{92}$ In this study, the distinction between idiopathic hypersomnia with and without long-sleep time was made according to American Academy of Sleep Medicine guidelines with idiopathic hypersomnia with long sleep time defined as excessive daytime sleepiness with an average total sleep time of $>10$ hours. ${ }^{1,92}$ Two other studies, however, found sleep paralysis to be far less common in idiopathic hypersomnia patients $(4 \%-10 \%){ }^{93,94}$ In summary, it is unclear whether sleep paralysis is clearly associated with sleep paralysis. No studies have directly contrasted rates of sleep paralysis in patients to matched controls. Given the diagnostic difficulty, and large heterogeneity that exists within patients, ${ }^{91}$ it may be that only a certain subgroup of patients are at a heightened risk of sleep paralysis. 


\section{Obstructive sleep apnea}

OSA is defined as repetitive episodes of complete or partial upper airway obstruction during sleep. In a study of OSA patients, $38 \%$ of the sample reported sleep paralysis. ${ }^{33}$ In a different study contrasting OSA patients with and without excessive sleepiness to healthy controls, a higher prevalence rate was found in OSA patients with excessive sleepiness (20\%) compared with OSA without excessive sleepiness $(0 \%)$ and healthy controls $(5 \%) .{ }^{95}$ The mechanism by which OSA increases the risk of sleep paralysis is still unclear. One possibility is that REM sleep may be fragmented due to airway restriction. ${ }^{84}$ Although apneas can occur at any point during sleep, the withdrawal of excitatory noradrenergic and serotonergic inputs to upper airway motor neurons during REM sleep reduces pharyngeal muscle activity and increases the propensity for upper airway collapse.$^{96}$ This means that the REM sleep of OSA patients is particularly vulnerable to disruption. ${ }^{84} \mathrm{Up}$ to $36 \%$ of OSA patients experience apneas primarily during REM sleep, and it would be interesting to investigate whether these patients are at an especially high risk of sleep paralysis..$^{84,97}$ In these patients, it is possible that the amount of REM sleep restriction is a significant predictor of sleep paralysis.

\section{Insomnia disorder}

It is unclear what is the relationship between sleep paralysis and insomnia disorder. Insomnia is a highly prevalent condition broadly involving difficulties going to and/or maintaining sleep. In a study of over 8,000 participants, where diagnosis was established via a computer program, those with severe sleep paralysis (defined as at least one episode per week) were found to have higher rates of insomnia (7.6\%) than those with less severe sleep paralysis (those who experience one episode a month or less) $(5.5 \%)$ or no sleep paralysis $(1.7 \%) .{ }^{98}$ However, in a multiple predictor model, insomnia was not a significant independent predictor of sleep paralysis. ${ }^{98}$ Using a similar method in a Spanish sample of 4,065 individuals, it was found that prevalence of sleep paralysis did not differ between those with and without an insomnia diagnosis. ${ }^{99}$ While little evidence has found significantly higher rates of sleep paralysis in those with an insomnia disorder, as seen previously some research has found that increased insomnia symptoms in otherwise healthy individuals does show a link to sleep paralysis.

In the most comprehensive examination of sleep paralysis in multiple sleep disorders to date, Ohayon et al surveyed sleep paralysis and potential predictors in 8,085 participants from Germany and Italy. ${ }^{98}$ Diagnosis of sleep disorders was carried out using lay investigators and a computer program (Sleep-EVAL). Using the International Classification of Sleep Disorders 1990 (ICSD-90) minimal criteria, participants who had experienced sleep paralysis were found to have higher rates of circadian rhythm disorder, psychophysiologic insomnia, OSA, periodic limb movement disorder, narcolepsy, confusional arousals, nocturnal leg cramps, sleep starts (also known as hypnic or hypnagogic jerks-sudden, brief, and strong contractions of the body or one or more body segments that occur when falling asleep), and sleep talking. ${ }^{98}$ However, in a multiple predictor model, where the various contributions of multiple different factors to the presence of sleep paralysis was assessed, the only sleep disorder to independently predict sleep paralysis was nocturnal leg cramps. ${ }^{98}$

In summary, it appears that sleep paralysis may be associated with a number of other sleep disorders. With the possible exception of narcolepsy, the exact nature of the relationship is unclear and comorbidity is not certain. Further research into sleep paralysis appearing in other sleep disorders is therefore warranted. This is especially true seeing as narcolepsy patients report sleep paralysis to be the most distressing aspect of the illness. ${ }^{84}$ How it may affect patients with other sleep disorders is an important avenue for future research to explore.

\section{Relevance to treatment}

The reviewed literature has clearly shown sleep quality to be linked with sleep paralysis. As such, any interventions designed to reduce/eliminate sleep paralysis need to take the patient's general sleep quality into account. A number of behavioral interventions for sleep paralysis have been proposed, but systematic testing is lacking. ${ }^{84,100,101}$ A cognitive behavioral therapy for sleep paralysis now exists and includes a screening for insomnia at initial assessment. ${ }^{84}$ Given the findings of this review, consistent checks as to the patient's sleep quality/insomnia symptoms throughout the treatment period should be performed. In addition, the co-occurrence of other sleep experiences such as nightmares and exploding head syndrome should be considered.

More generally, trying to maintain good sleep quality may be preventative to experiencing sleep paralysis. In a study that asked participants to describe methods used to prevent sleep paralysis episodes, changes to sleeping patterns were advocated by participants as being the most successful prevention technique. ${ }^{12}$ Techniques such as attaining a more regulated sleep schedule, stopping taking naps, and falling asleep in a different position were listed as specific changes participants made. ${ }^{12}$ As well as preventing episodes, being 
able to disrupt episodes as they occur may be an important coping strategy. In one study, $70 \%$ of participants reported trying to disrupt sleep paralysis episodes as they are happening compared with just $19 \%$ who tried to prevent episodes. ${ }^{12}$ Specifically, attempting to move extremities and other body parts such as the mouth or torso was the most frequently reported disruption techniques. ${ }^{12}$

Being able to disrupt episodes is likely to bring about a reduction in the fear/distress associated with sleep paralysis, as the individual becomes able to control and end the episode at will. As such, the reduced distress will mean that further reduction in sleep quality due to attempts to avoid sleeping will not occur. Lucid dreaming has been suggested as a potential treatment for nightmares, by allowing the individual to control the dream content thus being able to end the nightmare. ${ }^{102-104}$ Given that lucid dreaming appears to be associated with aspects of sleep paralysis that more typically are associated with positive experiences ${ }^{28}$ (ie, V-M hallucinations), ${ }^{9,80}$ learning to lucid dreaming techniques to help regain control of the episode or even transform sleep paralysis into a lucid dream may be an effective technique that warrants systematic testing. Indeed, the practice of transforming sleep paralysis episodes into more positive experiences such as lucid dreaming is actively sought after by many individuals and may be linked to spiritual experiences for some. ${ }^{105,106} \mathrm{It}$ is unfortunate that these potential positive aspects of sleep paralysis have generally been ignored in the literature, and future research should aim to tackle the question of whether transforming negative sleep paralysis episodes into positive experiences is feasible in all individuals.

\section{Limitations of the current literature and future directions}

Despite the number of studies being limited, the work carried out to date has provided great insights into how sleep paralysis is linked to other aspects of sleep. Results from survey studies have revealed a number of different aspects of sleep to be linked to sleep paralysis. Of note, many of these studies have used large sample sizes, which is a great strength to the work. However, there are still large gaps in the literature that future studies should aim to assess.

First, all of the current literature used cross-sectional designs, making it impossible to infer direction of effects. As such, it is still unknown whether poor sleep quality causes sleep paralysis or if sleep paralysis leads to poor sleep quality. As hypothesized previously, it is likely a bidirectional relationship. Factors such as genetics and particular stressors lead to a reduction in sleep quality, increasing the likelihood of sleep paralysis. In turn, if there is enough fear/associated distress, experiencing sleep paralysis may lead to a further decrease in sleep quality through maladaptive behaviors such as trying to stay awake to avoid an episode. Only in studies taking a longitudinal approach, perhaps using diary or experience sampling approaches, will the exact nature of the relationship be unraveled.

A second limitation of the literature to date is that the majority of research has been interested in simply the presence of lifetime episodes (ie, at least one episode in life) or the simple frequency of episodes. Associated features such as hallucinations, fear, and episode distress have been investigated far less frequently despite the fact that these aspects are likely of most relevance to individuals. Future research linking sleep paralysis to sleep quality should endeavor to stop using single-item measures of sleep paralysis occurrence, as has been the norm to date, in favor of more detailed questionnaires and clinical interviews. A few examples of such measures are the Waterloo Unusual Sleep Experiences Questionnaire, the Unusual Sleep Experiences Questionnaire, and the Fearful Isolated Sleep Paralysis Interview (FISPI). ${ }^{22,84,107,108}$ Currently, the FISPI is the only measure to tap into both the frequency of episodes and the associated features of hallucinations, fear, distress, and interference with waking life.

Objective studies of sleep paralysis are limited in their number, with only a handful of studies being conducted. This is partially due to the difficulty investigating sleep paralysis in a laboratory environment. One approach for future studies could be to make use of home monitoring devices such as portable PSG over an extended period of time (possibly as part of a longitudinal investigation of sleep paralysis), which could capture sporadic episodes of sleep paralysis as they occur. A second approach would be to use an intensive case study design, where a few individuals who do have nightly/multiple episodes per week spend multiple nights in the laboratory to maximize the number of sleep paralysis episodes that are recorded. Similar approaches have been successfully used to study sleep onset mentation and hypnagogic hallucinations. ${ }^{109,110}$

\section{Conclusion}

Our knowledge about sleep paralysis is still in its infancy. Nevertheless, good progress has been made in terms of investigating associations between sleep paralysis and other aspects of sleep. This research has shown sleep paralysis to be linked to more general sleep quality and insomnia symptoms. Furthermore, it appears that sleep paralysis does not necessarily occur in isolation, but frequently co-occurs with other 
sleep experiences such as nightmares and exploding head syndrome. Now, research needs to address the directionality of observed effects, in order to separate out the causes from the consequences of sleep paralysis.

\section{Acknowledgment}

The author thanks anonymous reviewers for their helpful and insightful comments.

\section{Disclosure}

The author reports no conflicts of interest in this work.

\section{References}

1. American Academy of Sleep Medicine. International Classificaiton of Sleep Disorders. 3rd ed. Darien, IL: Americian Academy of Sleep Medicine; 2014.

2. Fraigne JJ, Torontali ZA, Snow MB, Peever JH. REM Sleep at its Core - Circuits, Neurotransmitters, and Pathophysiology. Front Neurol. 2015;6(May):1-9.

3. Brooks PL, Peever JH. Identification of the transmitter and receptor mechanisms responsible for REM sleep paralysis. $J$ Neurosci. 2012;32(29):9785-9795.

4. Schenck CH, Lee SA, Bornemann MAC, Mahowald MW. Potentially lethal behaviors associated with rapid eye movement sleep behavior disorder: Review of the literature and forensic implications. J Forensic Sci. 2009;54(6):1475-1484.

5. Mutz J, Javadi A-H. Exploring the Neural Correlates of Dream Phenomenology and Altered States of Consciousness During Sleep. Neurosci Conscious. 2017;(June).

6. Mahowald MW, Schenck CH. Insights from studying human sleep disorders. Nature. 2005;437(7063):1279-1285.

7. Cheyne JA, Newby-Clark IR, Rueffer SD. Relations among hypnagogic and hypnopompic experiences associated with sleep paralysis. $J$ Sleep Res. 1999;8:313-317.

8. Cheyne JA, Rueffer SD, Newby-Clark IR. Hypnagogic and hypnopompic hallucinations during sleep paralysis: neurological and cultural construction of the night-mare. Conscious Cogn. 1999;8(3):319-337.

9. Cheyne JA. Sleep paralysis and the structure of waking-nightmare hallucinations. Dreaming. 2003;13(3):163-179.

10. Schredl M, Doll E. Emotions in Diary Dreams. Conscious Cogn. 1998;7(4):634-646.

11. Parker JD, Blackmore SJ. Comparing the content of sleep paralysis and dream reports. Dreaming. 2002;12(1):45-59.

12. Sharpless BA, Grom JL. Isolated sleep paralysis: Fear, prevention, and disruption. Behav Sleep Med. 2016;14(2):134-139.

13. Lišková M, Manková D, Bušková J. Could sleep paralysis be pleasant? Sleep Med. 2017;40(2017):e196-e197.

14. Pizza F, Moghadam KK, Franceschini C, et al. Rhythmic movements and sleep paralysis in narcolepsy with cataplexy: A video-polygraphic study. Sleep Med. 2010;11(4):423-425.

15. Takeuchi T, Miyasita A, Sasaki Y, Inugami M, Fukuda K. Isolated sleep paralysis elicited by sleep interruption. Sleep. 1992;15(3):217-225.

16. Buzzi G. False awakenings in light of the dream protoconsciousness theory: A study in lucid dreamers. Int $J$ Dream Res. 2011;4(2):110-116.

17. Nielsen TA, Zadra A. Nightmares and other common dream disturbances. In: Kryger MH, Roth T, Dement WC, eds. Principles and Practice of Sleep Medicine. 5th ed. Philadelphia: Elsevier Saunders; 2005:926-935.

18. Sharpless BA, Barber JP. Lifetime prevalence rates of sleep paralysis: a systematic review. Sleep Med Rev. 2011;15(5):311-315.
19. Molendijk ML, Montagne H, Bouachmir O, Alper Z, Bervoets J-P, Blom JD. Prevalence Rates of the Incubus Phenomenon: A Systematic Review and Meta-Analysis. Front Psychiatry. 2017;8:253.

20. Denis D, French CC, Gregory AM. A systematic review of variables associated with sleep paralysis. Sleep Med Rev. 2018;38:141-157.

21. Ramsawh HJ, Raffa SD, White KS, Barlow DH. Risk factors for isolated sleep paralysis in an African American sample: a preliminary study. Behav Ther. 2008;39(4):386-397.

22. Sharpless BA, McCarthy KS, Chambless DL, Milrod BL, Khalsa S-R, Barber JP. Isolated Sleep Paralysis and Fearful Isolated Sleep Paralysis in Outpatients With Panic Attacks. J Clin Psychol. 2010;66(12):1292-1307.

23. Hinton DE, Pich V, Chhean D, Pollack MH, McNally RJ. Sleep paralysis among Cambodian refugees: association with PTSD diagnosis and severity. Depress Anxiety. 2005;22(2):47-51.

24. Hinton DE, Pich V, Chhean D, Pollack MH. The Ghost Pushes You Down: Sleep Paralysis-Type Panic Attacks in a Khmer Refugee Population. Transcult Psychiatry. 2005;42(1):46-77.

25. Paradis C. M, Friedman S, Hatch M. Isolated sleep paralysis in African Americans with panic disorder. Cult Divers Ment Health. 1997;3(1):69-76.

26. Yeung A, Xu Y, Chang DF. Prevalence and Illness Beliefs of Sleep Paralysis among Chinese Psychiatric Patients in China and the United States. Transcult Psychiatry. 2005;42(1):135-145.

27. Denis D, French CC, Rowe R, et al. A twin and molecular genetics study of sleep paralysis and associated factors. J Sleep Res. 2015;24(4):438-446.

28. Denis D, Poerio GL. Terror and bliss? Commonalities and distinctions between sleep paralysis, lucid dreaming, and their associations with waking life experiences. J Sleep Res. 2017;26(1):38-47.

29. Szklo-Coxe M, Young T, Finn L, Mignot E. Depression: relationships to sleep paralysis and other sleep disturbances in a community sample. J Sleep Res. 2007;16(3):297-312.

30. Ma S, Wu T, Pi G. Sleep paralysis in Chinese adolescents: A representative survey. Sleep Biol Rhythms. 2014;12(1):46-52.

31. Munezawa T, Kaneita Y, Osaki Y, et al. Nightmare and sleep paralysis among Japanese adolescents: a nationwide representative survey. Sleep Med. 2011;12(1):56-64.

32. Buysse DJ, Reynolds C, Monk T, Berman S, Kupfer D. The Pittsburgh Sleep Quality Index (PSQI): A new instrument for psychiatric practice and research. Psychiatry Res. 1989;28(2):193-213.

33. Hsieh S-W, Lai C-L, Liu C-K, Lan S-H, Hsu C-Y. Isolated sleep paralysis linked to impaired nocturnal sleep quality and health-related quality of life in Chinese-Taiwanese patients with obstructive sleep apnea. Qual Life Res. 2010;19(9):1265-1272.

34. Munezawa T, Kaneita Y, Yokoyama E, Suzuki H, Ohida T. Epidemiological study of nightmare and sleep paralysis among Japanese adolescents. Sleep Biol Rhythms. 2009;7(3):201-210.

35. Denis D, French CC, Schneider MN, Gregory AM. Subjective sleeprelated variables in those who have and have not experienced sleep paralysis. J Sleep Res. 2018;27(5):e12650.

36. Okun ML, Kravitz HM, Sowers MF, Moul DE, Buysse DJ, Hall M. Psychometric evaluation of the insomnia symptom questionnaire: A self-report measure to identify chronic insomia. J Clin Sleep Med. 2009;5(1):41-51.

37. Espie CA, Kyle SD, Hames P, Gardani M, Fleming L, Cape J. The Sleep Condition Indicator: a clinical screening tool to evaluate insomnia disorder. BMJ Open. 2014;4(3):e004183.

38. Otsuka Y, Kaneita Y, Nakagome S, Jike M, Itani O, Ohida T. Nightmares and sleep paralysis among the general Japanese population: a nationwide representative survey. Sleep Biol Rhythms. 2018;16(2):187-195.

39. Bae K, Jin X, Maywood ES, Hastings MH, Reppert SM, Weaver DR. Differential functions of mPer1, mPer2, and mPer3 in the SCN circadian clock. Neuron. 2001;30(2):525-536.

40. Parsons MJ, Lester KJ, Barclay NL, et al. Polymorphisms in the circadian expressed genes PER3 and ARNTL2 are associated with diurnal preference and GN 33 with sleep measures. J Sleep Res. March 2014 
41. van der Kloet D, Merckelbach H, Giesbrecht T, Lynn SJ. Fragmented Sleep, Fragmented Mind: The Role of Sleep in Dissociative Symptoms. Perspect Psychol Sci. 2012;7(2):159-175.

42. Gregory AM, Buysse DJ, Willis TA, et al. Associations between sleep quality and anxiety and depression symptoms in a sample of young adult twins and siblings. $J$ Psychosom Res. 2011;71(4):250-255.

43. Barclay NL, Eley TC, Rijsdijk F V., Gregory AM. Dependent negative life events and sleep quality: An examination of gene-environment interplay. Sleep Med. 2011;12(4):403-409.

44. Baglioni C, Battagliese G, Feige B, et al. Insomnia as a predictor of depression: A meta-analytic evaluation of longitudinal epidemiological studies. J Affect Disord. 2011;135(1-3):10-19.

45. Hayes AF. Introduction to Mediation, Moderation, and Conditional Process Analysis. Guilford Press; 2013.

46. Cheyne JA, Pennycook G. Sleep Paralysis Postepisode Distress: Modeling Potential Effects of Episode Characteristics, General Psychological Distress, Beliefs, and Cognitive Style. Clin Psychol Sci. 2013;1(2):135-148.

47. Cheyne JA. Situational factors affecting sleep paralysis and associated hallucinations: position and timing effects. $J$ Sleep Res. 2002;11(2):169-177.

48. Fukuda K, Ogilvie RD, Chilcott L, Vendittelli A-M, Takeuchi T. The prevalence of sleep paralysis among Canadian and Japanese college students. Dreaming. 1998;8(2):59-66.

49. Sharpless BA. Exploding head syndrome is common in college students. J Sleep Res. 2015;24(4):447-449.

50. Evans RW. Exploding head syndrome followed by sleep paralysis: A rare migraine aura. Headache. 2006;46(4):682-683.

51. Spanos NP, McNulty SA, DuBreuil SC, Pires M, Faith Burgess M. The frequency and correlates of sleep paralysis in a university sample. $J$ Res Pers. 1995;29:285-305.

52. Germain A, Hall M, Krakow B, Katherine Shear M, Buysse DJ. A brief Sleep Scale for Posttraumatic Stress Disorder: Pittsburgh Sleep Quality Index Addendum for PTSD. J Anxiety Disord. 2005;19(2):233-244.

53. Emslie K. Hallucinogenic nights. Aeon. https://aeon.co/essays/theterror-and-the-bliss-of-sleep-paralysis. Published 2014. Accessed April 2, 2016.

54. Schredl M, Erlacher D. Lucid dreaming frequency and personality. Pers Individ Dif. 2004;37:1463-1473.

55. Voss U, Schermelleh-Engel K, Windt J, Frenzel C, Hobson A. Measuring consciousness in dreams: the lucidity and consciousness in dreams scale. Conscious Cogn. 2013;22(1):8-21.

56. Solomonova E, Nielsen T, Stenstrom P. Lucid dreaming is associated with sleep paralysis but not nightmaries. In: 4th Conference of the Canadian Sleep Society. Toronto, ON; 2009.

57. Watson D. Dissociations of the night: Individual differences in sleeprelated experiences and their relation to dissociation and schizotypy. J Abnorm Psychol. 2001;110(4):526-535.

58. American Psychiatric Association. Diagnostic and Statistical Manual of Mental Health Disorders. 5th ed. Washington, DC: American Psychiatric Association; 2013.

59. Abrams MP, Mulligan AD, Carleton RN, Asmundson GJG. Prevalence and correlates of sleep paralysis in adults reporting childhood sexual abuse. J Anxiety Disord. 2008;22(8):1535-1541.

60. McNally RJ, Clancy SA. Sleep paralysis in adults reporting repressed, recovered, or continuous memories of childhood sexual abuse. $J$ Anxiety Disord. 2005;19(5):595-602.

61. Lišková M, Janečková $\mathrm{D}$, Kráčmarová LK, Mladá K, Bušková J. The occurrence and predictive factors of sleep paralysis in university students. Neuropsychiatr Dis Treat. 2016;12:2957-2962.

62. Giesbrecht T, Smeets T, Leppink J, Jelicic M, Merckelbach H. Acute Dissociation After 1 Night of Sleep Loss. J Abnorm Psychol. 2007;116(3):599-606.

63. van der Kloet D, Giesbrecht T, Lynn SJ, Merckelbach H, de Zutter A. Sleep normalization and decrease in dissociative experiences: Evaluation in an inpatient sample. JAbnorm Psychol. 2012;121(1):140-150.
64. Walther BW, Schulz H. Recurrent Isolated Sleep Paralysis: Polysomnographic and Clinical Findings. Somnologie. 2004;8(2):53-60.

65. Takeuchi T, Fukuda K, Sasaki Y, Inugami M, Murphy TI. Factors related to the occurrence of isolated sleep paralysis elicited during a multi-phasic sleep-wake schedule. Sleep. 2002;25(1):89-96.

66. Terzaghi M, Ratti PL, Manni F, Manni R. Sleep paralysis in narcolepsy: more than just a motor dissociative phenomenon? Neurol Sci. 2012;33(1):169-172.

67. Sharpless BA, Denis D. Recurrent isolated sleep paralysis. In: Stein J, ed. Reference Module in Neuroscience and Biobehavioral Psychology. New York, NY: Elsevier; 2017:1-5.

68. Cheyne JA. Recurrent isolated sleep paralysis. In: Thorpy MJ, Pazzi G, eds. The Parasomnias and Other Sleep-Related Movement Disorders. New York, NY: Cambridge University Press; 2010:142-152.

69. Hobson JA. REM sleep and dreaming: towards a theory of protoconsciousness. Nat Rev Neurosci. 2009;10(11):803-813.

70. Fox KCR, Nijeboer S, Solomonova E, Domhoff GW, Christoff $\mathrm{K}$. Dreaming as mind wandering: evidence from functional neuroimaging and first-person content reports. Front Hum Neurosci. 2013;7(July):412.

71. Voss U, Holzmann R, Tuin I, Hobson JA. Lucid dreaming: a state of consciousness with features of both waking and non-lucid dreaming. Sleep. 2009;32(9):1191-1200.

72. Voss U, Holzmann R, Hobson A, et al. Induction of self awareness in dreams through frontal low current stimulation of gamma activity. Nat Neurosci. 2014;17(6):810-812.

73. Dresler M, Wehrle R, Spoormaker VI, et al. Neural Correlates of Dream Lucidity Obtained from Contrasting Lucid versus Non-Lucid REM Sleep: A Combined EEG/fMRI Case Study. Sleep. 2012:4-7.

74. Adolphs R. The biology of fear. Curr Biol. 2013;23(2):R79-R93.

75. Marek R, Strobel C, Bredy TW, Sah P. The amygdala and medial prefrontal cortex: Partners in the fear circuit. J Physiol. 2013;591(10):2381-2391.

76. Nielsen T, Levin R. Nightmares: A new neurocognitive model. Sleep Med Rev. 2007;11(4):295-310.

77. Nielsen T. The stress acceleration hypothesis of nightmares. Front Neurol. 2017;8(JUN).

78. Cheyne JA, Girard TA. Paranoid delusions and threatening hallucinations: a prospective study of sleep paralysis experiences. Conscious Cogn. 2007;16(4):959-974.

79. Guterstam A, Björnsdotter M, Gentile G, Ehrsson HH. Posterior cingulate cortex integrates the senses of self-location and body ownership. Curr Biol. 2015;25(11):1416-1425.

80. Cheyne JA, Girard TA. The body unbound: vestibular-motor hallucinations and out-of-body experiences. Cortex. 2009;45(2):201-215.

81. Blanke O, Mohr C, Michel CM, et al. Linking Out-of-Body Experience and Self Processing to Mental Own-Body Imagery at the Temporoparietal Junction. J Neurosci. 2005;25(3):550-557.

82. Blanke O, Arzy S. The Out-of-Body Experience: Disturbed Self-Processing at the Temporo-Parietal Junction. Neurosci. 2005;11(1):16-24.

83. Scammell TE. The neurobiology, diagnosis, and treatment of narcolepsy. Ann Neurol. 2003;53(2):154-166.

84. Sharpless BA, Doghramji K. Sleep Paralysis: Historical, Psychological, and Medical Perspectives. New York, NY: Oxford University Press; 2015.

85. Leu-Semenescu S, De Cock VC, Le Masson VD, et al. Hallucinations in narcolepsy with and without cataplexy: Contrasts with Parkinson's disease. Sleep Med. 2011;12(5):497-504.

86. Bassetti C. Idiopathic hypersomnia. A series of 42 patients. Brain. 1997;120(8):1423-1435.

87. Mahlios J, De la Herrán-Arita AK, Mignot E. The autoimmune basis of narcolepsy. Curr Opin Neurobiol. 2013;23(5):767-773.

88. Andlauer O, Moore HE, Hong S-C, et al. Predictors of Hypocretin (Orexin) Deficiency in Narcolepsy Without Cataplexy. Sleep. 2012;35(9):1247-1255.

89. Dahlitz M, Parkes J. D. Sleep paralysis. Lancet. 1993;341(8842):406-407. 
90. Okun ML, Lin L, Pelin Z, Hong S, Mignot E. Clinical aspects of narcolepsy-cataplexy across ethnic groups. Sleep. 2002;25(1):27-35.

91. Montplaisir J, Fantini L. Idiopathic hypersomnia: A diagnostic dilemma. A commentary on "Idiopathic hypersomnia"). Sleep Med Rev. 2001;5(5):361-362.

92. Vernet C, Arnulf I. Idiopathic hypersomnia with and without long sleep time: a controlled series of 75 patients. Sleep. 2009;32(6):753-759.

93. Ali M, Auger RR, Slocumb NL, Morgenthaler TI. Idiopathic hypersomnia: Clinical features and response to treatment. J Clin Sleep Med. 2009;5(6):562-568.

94. Anderson KN, Pilsworth S, Sharples LD, Smith IE, Shneerson JM. Idiopathic hypersomnia: A study of 77 cases. Sleep. 2007;30(10):1274-1281.

95. Vernet C, Redolfi S, Attali V, et al. Residual sleepiness in obstructive sleep apnoea: Phenotype and related symptoms. Eur Respir J. 2011;38(1):98-105.

96. Fenik VB, Davies RO, Kubin L. REM sleep-like atonia of hypoglossal (XII) motoneurons is caused by loss of noradrenergic and serotonergic inputs. Am J Respir Crit Care Med. 2005;172(10):1322-1330.

97. Mokhlesi B, Punjabi NM. "REM-related" Obstructive Sleep Apnea: An Epiphenomenon or a Clinically Important Entity? Sleep. 2012;35(1):5-7.

98. Ohayon MM, Zulley J, Guilleminault C, Smirne S. Prevalence and pathologic associations of sleep paralysis in the general population. Neurology. 1999;52(6):1194-1194.

99. Ohayon MM, Sagales T. Prevalence of insomnia and sleep characteristics in the general population of Spain. Sleep Med. 2010;11(10):1010-1018.

100. Sharpless BA. A clinician's guide to isolated sleep paralysis. Neuropsychiatr Dis Treat. 2016; Volume 12(August):1761-1767.
101. Jalal B. How to Make the Ghosts in my Bedroom Disappear ? FocusedAttention Meditation Combined with Muscle Relaxation ( MR Therapy ) - A Direct Treatment Intervention for Sleep Paralysis How to Make the Ghosts in my Bedroom Disappear ? Focused-Attention Meditation. Front Psychol. 2016;(February).

102. Spoormaker VI, Van Den Bout J. Lucid dreaming treatment for nightmares: A pilot study. Psychother Psychosom. 2006;75(6):389-394.

103. Spoormaker VI, Bout J Van Den, Meijer EJG. Lucid Dreaming Treatment for Nightmares : ASeries of Cases. Dreaming. 2003;13(3):181-186.

104. Zadra AL, Pihl RO. Lucid dreaming as a treatment for recurrent nightmares. Psychother Psychosom. 1997;66:50-55.

105. Solomonova E. Sleep Paralysis: phenomenology, neurophysiology and treatment. In: Fox K, Christoff K, eds. The Oxford Handbook of Spontaneous Thought: Mind-Wandering, Creativity, Dreaming, and Clinical Conditions. Oxford University Press; 2018:435-456.

106. Hufford DJ. Sleep Paralysis as Spiritual Experience. Transcult Psychiatry. 2005;42(1):11-45.

107. Cheyne JA. Waterloo unusual sleep experiences questionnaire-VIIIa.

108. Paradis C, Friedman S, Hinton DE, McNally RJ, Solomon LZ, Lyons KA. The assessment of the phenomenology of sleep paralysis: The unusual sleep experiences questionnaire (USEQ). CNS Neurosci Ther. 2009; 15:220-226.

109. Noreika V, Canales-Johnson A, Koh J, Taylor M, Massey I, Bekinschtein TA. Intrusions of a drowsy mind: Neural markers of phenomenological unpredictability. Front Psychol. 2015;6(MAR):1-10.

110. Stenstrom P, Fox K, Solomonova E, Nielsen T. Mentation during sleep onset theta bursts in a trained participant: A role for NREM stage 1 sleep in memory processing? Int J Dream Res. 2012;5(1):37-46.
Nature and Science of Sleep

\section{Publish your work in this journal}

Nature and Science of Sleep is an international, peer-reviewed, open access journal covering all aspects of sleep science and sleep medicine, including the neurophysiology and functions of sleep, the genetics of sleep, sleep and society, biological rhythms, dreaming, sleep disorders and therapy, and strategies to optimize healthy sleep. The manuscript

\section{Dovepress}

management system is completely online and includes a very quick and fair peer-review system, which is all easy to use. Visit http://www. dovepress.com/testimonials.php to read real quotes from published authors. 